\title{
An Existence Theorem of Solutions for the System of Generalized Vector Quasi-Variational-Like Inequalities
}

\author{
Shamshad Husain ${ }^{1}$, Sanjeev Gupta ${ }^{1 *}$, Vishnu Narayan Mishra ${ }^{2}$ \\ ${ }^{1}$ Department of Applied Mathematics, Faculty of Engineering \& Technology, Aligarh Muslim University, Aligarh, India \\ ${ }^{2}$ Department of Applied Mathematics \& Humanities, Sardar Vallabhbhai National Institute of Technology, Surat, India \\ Email: s_husain68@yahoo.com, "guptasanmp@gmail.com, vishnunarayanmishra@gmail.com
}

Received May 14, 2012; revised June 20, 2012; accepted July 8, 2012

Copyright (C) 2013 Shamshad Husain et al. This is an open access article distributed under the Creative Commons Attribution License, which permits unrestricted use, distribution, and reproduction in any medium, provided the original work is properly cited.

\begin{abstract}
In this paper, we introduce and study the system of generalized vector quasi-variational-like inequalities in Hausdorff topological vector spaces, which include the system of vector quasi-variational-like inequalities, the system of vector variational-like inequalities, the system of vector quasi-variational inequalities, and several other systems as special cases. Moreover, a number of C-diagonal quasiconvexity properties are proposed for set-valued maps, which are natural generalizations of the g-diagonal quasiconvexity for real functions. Together with an application of continuous selection and fixed-point theorems, these conditions enable us to prove unified existence results of solutions for the system of generalized vector quasi-variational-like inequalities. The results of this paper can be seen as extensions and generalizations of several known results in the literature.
\end{abstract}

Keywords: The System of Generalized Vector Quasi-Variational-Like Inequalities; Fixed Point Theorem; Open Lower Section; Upper Semicontinuous; C-Diagonal Quasiconvexity

\section{Introduction and Formulation}

In recent years, the system of generalized vector quasivariational-like inequality, which is a unified model for the system of vector quasi-variational-like inequalities, the system of vector variational-like inequalities, the system of vector variational inequalities, the system of vector equilibrium problems and the system of variational inequalities etc., has been studied (see [1-18] and references therein).

In this paper, we consider the systems of four kinds of generalized vector quasi-variational-like inequalities with set-valued mappings and discuss the existence of its solutions in locally convex topological vector space (1.c.s. in short), motivated and inspired by the recent works of Peng [1] and Ansari et al. [2].

Throughout this paper, unless otherwise specified, assume that $I$ be an index set. For each $i \in I$, let $Z_{i}$ be a locally convex topological vector space (l.c.s., in short) and $K_{i}$ be a nonempty convex subset of Hausdorff topological vector space (t.v.s., in short) $E_{i}$. Let $Y_{i}$ be a subset of continuous function space $L\left(E_{i}, Z_{i}\right)$ from $E_{i}$ into $Z_{i}$, where $L\left(E_{i}, Z_{i}\right)$ is equipped with a $\sigma$ -

${ }^{*}$ Corresponding author. topology. Let int $A$ and $\operatorname{co} A$ denote the interior and convex hull of a set $A$ respectively. Let $C_{i}: K \rightarrow 2^{Z_{i}}$ be a set-valued mapping such that $\operatorname{int} C_{i}(x) \neq \varnothing$ for each $x \in K$. Denote that $K=\prod_{i \in I} K_{i}$ and $E=\prod_{i \in I} E_{i}$.

For each $i \in I$, let $\eta_{i}: K_{i} \times K_{i} \rightarrow E_{i}$ be a vectorvalued mapping, $G_{i}: L(E, Z) \rightarrow 2^{L\left(E_{i}, Z_{i}\right)}$,

$S_{i}: K \times K \rightarrow 2^{Z_{i}}, T_{i}: K \rightarrow 2^{Y_{i}}$ and $D_{i}: K \rightarrow 2^{K_{i}}$ be four set-valued mappings. Then,

1) Strong type I system of generalized vector quasivariational-like inequalities which is to find $(\bar{x}, \bar{t}) \in K \times Y$ such that $\bar{x}_{i} \in D_{i}(\bar{x}), \bar{t}_{i} \in T_{i}(\bar{x})$ and $\left\langle G_{i} \bar{t}_{i}, \eta_{i}\left(y_{i}, \bar{x}_{i}\right)\right\rangle+S_{i}\left(\bar{x}_{i}, y_{i}\right) \subseteq C_{i}(\bar{x}), \forall y_{i} \in D_{i}(\bar{x}),(1.1)$

2) Strong type II system of generalized vector quasivariational-like inequalities which is to find $(\bar{x}, \bar{t}) \in K \times Y$ such that $\bar{x}_{i} \in D_{i}(\bar{x}), \bar{t}_{i} \in T_{i}(\bar{x})$ and

$$
\begin{aligned}
& \left\{\left\langle G_{i} \bar{t}_{i}, \eta_{i}\left(y_{i}, \bar{x}_{i}\right)\right\rangle+S_{i}\left(\bar{x}_{i}, y_{i}\right)\right\} \\
& \cap C_{i}(\bar{x}) \neq \varnothing, \forall y_{i} \in D_{i}(\bar{x}),
\end{aligned}
$$

3) Weak type I system of generalized vector quasivariational-like inequalities which is to find $(\bar{x}, \bar{t}) \in K \times Y$ such that $\bar{x}_{i} \in D_{i}(\bar{x}), \bar{t}_{i} \in T_{i}(\bar{x})$ and 


$$
\begin{aligned}
& \left\{\left\langle G_{i} \bar{t}_{i}, \eta_{i}\left(y_{i}, \bar{x}_{i}\right)\right\rangle+S_{i}\left(\bar{x}_{i}, y_{i}\right)\right\} \cap-\operatorname{int} C_{i}(\bar{x}) \neq \varnothing, \\
& \forall y_{i} \in D_{i}(\bar{x}),
\end{aligned}
$$

4) Weak type II system of generalized vector quasivariational-like inequalities which is to find $(\bar{x}, \bar{t}) \in K \times Y$ such that $\bar{x}_{i} \in D_{i}(\bar{x}), \bar{t}_{i} \in T_{i}(\bar{x})$ and

$$
\left\langle G_{i} \bar{t}_{i}, \eta_{i}\left(y_{i}, \bar{x}_{i}\right)\right\rangle+S_{i}\left(\bar{x}_{i}, y_{i}\right) \not \subseteq-\operatorname{int} C_{i}(\bar{x}), \forall y_{i} \in D_{i}(\bar{x}),
$$

where $\langle l, x\rangle$ denotes the evaluation of $l \in L(E, Z)$ at $x \in E$. By the corollary of the Schaefer [3], $L(E, Z)$ becomes a 1.c.s.. By Ding and Tarafdar [4], the bilinear map $\langle\cdot, \cdot\rangle: L(K, Z) \times K \rightarrow Z$ is continuous.

The following problems are the special cases of above four kinds of systems of generalized vector quasi-variational-like inequalities.

The above system of generalized vector quasi-variational-like inequalities encompass many models of system of variational inequalities. The following problems are the special cases of problem (1.4).

1) If for each $i \in I$, let $G_{i}$ be an identity mapping, $S_{i} \equiv 0$, problem (1.4) reduces to the system of generalized quasi-variational-like inequalities of finding $\bar{x} \in K$ such that for each $i \in I, \bar{x}_{i} \in D_{i}(\bar{x})$ and

$$
\forall y_{i} \in D_{i}(\bar{x}), \exists \overline{t_{i}} \in T_{i}(\bar{x}):\left\langle\overline{t_{i}}, \eta_{i}\left(y_{i}, \bar{x}_{i}\right)\right\rangle \notin-\operatorname{int} C_{i}(\bar{x}),
$$

which was introduced and studied by Peng [1].

2) If for each $i \in I$, let $G_{i}$ be an identity mapping, $S_{i} \equiv 0$ and $D_{i}(x)=K_{i}$, problem (1.5) reduces to the system of generalized variational-like inequalities of finding $\bar{x} \in K$ such that for each $i \in I, \bar{x}_{i} \in K_{i}$ and

$$
\forall y_{i} \in K_{i}, \exists \bar{t}_{i} \in T_{i}(\bar{x}):\left\langle\bar{t}_{i}, \eta_{i}\left(y_{i}, \bar{x}_{i}\right)\right\rangle \notin-\operatorname{int} C_{i}(\bar{x}) .
$$

In addition, let $Z_{i}=\mathbb{R}$ and let $C_{i}(x)=\mathbb{R}^{+}=\{r \in \mathbb{R} \mid r \geq 0\}$ for all $x \in K$, then problem (1.5) reduces to the system of generalized vector quasi-variational inequalities studied by Ansari and Yao [5].

3) If for each $i \in I, G_{i}$ be an identity mapping, $S_{i} \equiv 0, \eta_{i}\left(y_{i}, \bar{x}_{i}\right)=y_{i}-\bar{x}_{i}$ and $D_{i}(x)=K_{i}$, then problem (1.5) reduces to the system of generalized vector variational inequalities of finding $\bar{x} \in K$ such that for each $i \in I, \bar{x}_{i} \in K_{i}$ and

$$
\forall y_{i} \in K_{i}, \exists \bar{t}_{i} \in T_{i}(\bar{x}):\left\langle\bar{t}_{i}, y_{i}-\bar{x}_{i}\right\rangle \notin-\operatorname{int} C_{i}(\bar{x}) .
$$

4) If $I=\{1\}$, problem (1.4) reduces to generalized vector quasi-variational-like inequalities of finding $\bar{x} \in K$ such that $\bar{x} \in D(\bar{x})$ and

$$
\langle G \bar{t}, \eta(y, \bar{x})\rangle+S(\bar{x}, y) \nsubseteq \neq-\operatorname{int} C(\bar{x}), \forall y_{i} \in K,
$$

such type of problem studied in [6-10].
5) If $I=\{1\}$ and $\eta(y, \bar{x})=y-\bar{x}, \quad T$ is single valued mapping, $G$ be an identity mapping, $S \equiv 0$, and $C(x)=\mathbb{R}^{+}$for all $x \in K$, then problem (1.4) reduces to classical variational inequality problem of finding $\bar{x} \in K$ such that $\bar{x} \in D(\bar{x})$ and

$$
\forall y \in D(\bar{x}), \exists \bar{t} \in T(\bar{x}):\langle T(\bar{x}),(y-\bar{x})\rangle \notin-\operatorname{int} C(\bar{x}),
$$

which was introduced and studied by Hartman and Stampacchia [11].

\section{Preliminaries}

Definition 2.1. [12] Let $E$ and $Z$ be two t.v.s. and $K$ be a convex subset of t.v.s. $E$. Let $C: K \rightarrow 2^{Z}$ and $\theta: K \times K \rightarrow 2^{Z}$ be two set-valued mappings. Assume given any finite subset $\Lambda=\left\{x_{1}, x_{2}, \cdots, x_{n}\right\}$ in $K$, any $x=\sum_{i=1}^{n} \alpha_{i} x_{i}$, with $\alpha_{i} \geq 0$ for $i=1, \cdots, n$, and $\sum_{i=1}^{n} \alpha_{i}=1$. Then, 1) $\theta$ is said to be strong Type I C-diagonally quasiconvex (SIC-DQC, in short) in the second argument if for some $x_{i} \in \Lambda$,

$$
\theta\left(x, x_{i}\right) \subseteq C(x)
$$

2) $\theta$ is said to be strong Type II C-diagonally quasiconvex (SIIC-DQC, in short) in the second argument if for some $x_{i} \in \Lambda$,

$$
\theta\left(x, x_{i}\right) \cap C(x) \neq \varnothing
$$

3) $\theta$ is said to be weak Type I C-diagonally quasiconvex (WIC-DQC, in short) in the second argument if for some $x_{i} \in \Lambda$,

$$
\theta\left(x, x_{i}\right) \cap-\operatorname{int} C(x) \neq C(x) ;
$$

4) $\theta$ is said to be weak Type II C-diagonally quasiconvex (WIIC-DQC, in short) in the second argument if for some $x_{i} \in \Lambda$,

$$
\theta\left(x, x_{i}\right) \nsubseteq \neq-\operatorname{int} C(x) .
$$

It is easy to verify that the following proposition, 1) SIC-DQC implies SIIC-DQC; 2) SIIC-DQC implies WIC-DQC; 3) WIC-DQC implies WIIC-DQC. The converse is not true. Following example shows that the con0 verse is not true.

Example 2.1. Let $E=Z=\mathbb{R}$ and $\varphi\left(x_{1}, x_{2}\right)=\operatorname{co}\left\{x_{1}, x_{2}\right\}$.

1) If $C(x)=[x+\epsilon,+\infty)$. Then $\varphi$ is SIIC-DQC, but it is not SIC-DQC.

2) If $-\operatorname{int} C(x)=(-\infty, x+\epsilon)$. Then $\varphi$ is WIICDQC, but it is not WIC-DQC.

Definition 2.2. [13] Let $E$ and $Z$ be two t.v.s. and $K$ be a convex subset of t.v.s. $E$. A mapping $\theta: K \times K \rightarrow\left(2^{Z}\right) Z$ is called (generalized) vector 0 - 
diagonally convex if for any finite subset

$$
\begin{aligned}
& \Lambda=\left\{x_{1}, x_{2}, \cdots, x_{n}\right\} \text { of } K \text { and any } x=\sum_{i=1}^{n} \alpha_{i} x_{i} \text { with } \\
& \alpha_{i} \geq 0 \text { for } i=1, \cdots, n \text {, and } \sum_{i=1}^{n} \alpha_{i}=1 \\
& \qquad \sum_{i=1}^{n} \alpha_{i} \theta\left(x, x_{i}\right)(\nsubseteq \notin) \notin-\operatorname{int} C(x) .
\end{aligned}
$$

Definition 2.3. [14] Let $X$ and $Y$ be two topological spaces and $T: X \rightarrow 2^{Y}$ be a set-valued mapping. Then,

1) $T$ is said to have open lower sections if the set $T^{-1}(y)=\{x \in X: y \in T(x)\}$ is open in $X$ for every $y \in Y$;

2) $T$ is said to be upper semicontinuous (u.s.c., in short) if for each $x_{o} \in X$ and each open set $U$ in $Y$ with $T\left(x_{o}\right) \subset U$, there exists an open neighborhood $V$ of $x_{o}$ in $X$ such that $T(x) \subset U$ for each $x \in V$;

3) $T$ is said to be lower semicontinuous (1.s.c., in short) if for each $x_{o} \in X$ and each open set $U$ in $Y$ with $T\left(x_{o}\right) \cap U \neq \varnothing$, there exists an open neighborhood $V$ of $x_{o}$ in $X$ such that $T(x) \cap U \neq \varnothing$ for each $x \in V$;

4) $T$ is said to be continuous if it is both upper and lower semicontinuous;

5) $T$ is said to be closed if for any net $\left\{x^{\circ}\right\}$ in $X$ such that $x^{o} \rightarrow x^{*}$ and any net $\left\{y^{o}\right\}$ in $B$ such that $y^{o} \rightarrow y^{*}$ and $y^{o} \in T\left(x^{o}\right)$ for any $o$, we have $y^{*} \in T\left(x^{*}\right)$.

Lemma 2.1. [15] Let $X$ and $Y$ be two topological spaces. If $T: X \rightarrow 2^{Y}$ is u.s.c. set-valued mapping with closed values, then $T$ is closed.

Lemma 2.2. [16] Let $X$ and $Y$ be two topological spaces and $T: X \rightarrow 2^{Y}$ is u.s.c. mapping with compact values. Suppose $\left\{x^{\circ}\right\}$ is a net in $X$ such that $x^{o} \rightarrow x^{*}$. If $y^{o} \in T\left(x^{o}\right)$ for each $O$, then there are a $y^{*} \in T\left(x^{*}\right)$ and a subnet $\left\{y^{n}\right\}$ of $\left\{y^{o}\right\}$ such that $y^{n} \rightarrow y^{*}$.

Lemma 2.3. [17] Let $X$ and $Y$ be two topological spaces. Suppose that $T: X \rightarrow 2^{Y}$ and $K: X \rightarrow 2^{Y}$ are set-valued mappings having open lower sections, then

1) A set-valued mapping $F: X \rightarrow 2^{Y}$ defined by, for each $x \in X, F(x)=\operatorname{coT}(x)$ has open lower sections;

2) A set-valued mapping $J: X \rightarrow 2^{Y}$ defined by, for each $x \in X, J(x)=T(x) \cap K(x)$ has open lower sections.

For each $i \in I, E_{i}$ a Hausdorff t.v.s. Let $\left\{K_{i}\right\}$ be a family of nonempty compact convex subsets with each $K_{i}$ in $E_{i}$. Let $K=\prod_{i \in I} K_{i}$ and $E=\prod_{i \in I} E_{i}$. The following system of fixed-point theorem is needed in this paper.

Lemma 2.4. [18] For each $i \in I$, let $T_{i}: K \rightarrow 2^{K_{i}}$ be a set-valued mapping. Assume that the following conditions hold.

1) For each $i \in I, T_{i}$ is convex set-valued mapping;

2) $K=\bigcup\left\{\operatorname{int} T_{i}^{-1}\left(x_{i}\right): x_{i} \in K_{i}\right\}$.

Then there exist $\bar{x} \in K$ such that $\bar{x} \in T(\bar{x})=\prod_{i \in I} T_{i}(\bar{x})$, that is, $\bar{x}_{i} \in T_{i}(\bar{x})$ for each $i \in I$, where $\bar{x}_{i}$ is the projection of $\bar{x}$ onto $K_{i}$.

\section{Main Results}

Theorem 3.1. For each $i \in I$, let $Z_{i}$ be a 1.c.s., $K_{i}$ a nonempty compact convex subset of Hausdorff t.v.s. $E_{i}$, $Y_{i}$ a nonempty compact convex subset of $L\left(E_{i}, Z_{i}\right)$, which is equipped with a $\sigma$-topology. For each $i \in I$, assume that the following conditions are satisfied.

1) $D_{i}: K \rightarrow 2^{K_{i}}$ and $T_{i}: K \rightarrow 2^{Y_{i}}$ are two nonempty convex set-valued mappings and have open lower sections;

2) For each $t_{i} \in Y_{i}$ and $x_{i} \in \operatorname{co} \Lambda_{i}$, the mapping $\left\langle G_{i} t_{i}, \eta_{i}\left(., x_{i}\right)\right\rangle+S_{i}\left(x_{i},.\right): K \rightarrow 2^{Z_{i}}$ is WIIC-DQC;

3) For each $y_{i} \in K_{i}$, the set

$\left\{(x, t) \in K \times Y:\left\langle G_{i} t_{i}, \eta_{i}\left(y_{i}, x_{i}\right)\right\rangle+S_{i}\left(x_{i}, y_{i}\right) \subseteq-\operatorname{int} C_{i}(x)\right\}$ is open.

Then there exist $\bar{x}_{i} \in D_{i}(\bar{x})$ and $\bar{t}_{i} \in T_{i}(\bar{x})$ such that

$$
\begin{aligned}
& \left\langle G_{i} \bar{t}_{i}, \eta_{i}\left(y_{i}, \bar{x}_{i}\right)\right\rangle+S_{i}\left(\bar{x}_{i}, y_{i}\right) \nsubseteq-\operatorname{int} C_{i}(\bar{x}), \\
& \forall y_{i} \in D_{i}(\bar{x}) .
\end{aligned}
$$

Proof. Define a set-valued mapping $P_{i}: K \times Y \rightarrow 2^{K_{i}}$ by

$$
\begin{aligned}
P_{i}(x, t)= & \left\{y_{i} \in K_{i}:\left\langle G_{i} t_{i}, \eta_{i}\left(y_{i}, x_{i}\right)\right\rangle\right. \\
& \left.+S_{i}\left(x_{i}, y_{i}\right) \subseteq-\operatorname{int} C_{i}(x)\right\}, \\
\forall(x, t) \in & K \times Y .
\end{aligned}
$$

We first prove that $x_{i} \notin \operatorname{co}\left(P_{i}(x, t)\right)$ for all $(x, t) \in K \times Y$. To see this, suppose, by way of contradiction, that there exist some $i \in I$ and some point $(\bar{x}, \bar{t}) \in K \times Y$ such that $\bar{x}_{i} \in \operatorname{co}\left(P_{i}(\bar{x}, \bar{t})\right)$. Then, there exist finite points $y_{i_{1}}, y_{i_{2}}, \cdots, y_{i_{n}}$ in $K_{i}$ and $\alpha_{j} \geq 0$ with $\sum_{j=1}^{n} \alpha_{j}=1$ such that $\bar{x}_{i}=\sum_{j=1}^{n} \alpha_{j} y_{i_{j}}$ and $y_{i_{j}} \in P_{i}(\bar{x}, \bar{t})$ for all $j=1, \cdots, n$ such that $\left\langle G_{i} \bar{t}_{i}, \eta_{i}\left(y_{i_{j}}, \bar{x}_{i}\right)\right\rangle+S_{i}\left(\bar{x}_{i}, y_{i_{j}}\right) \subseteq-\operatorname{int} C_{i}(\bar{x}), \quad j=1, \cdots, n$, which contradicts the hypothesis 2$)$. Hence, $x_{i} \notin \operatorname{co}\left(P_{i}(x, t)\right)$.

By hypothesis 3), for each $i \in I$ and each $y_{i} \in K_{i}$, we known that 


$$
\begin{aligned}
Q_{i}^{-1}\left(y_{i}\right)= & \left\{(x, t) \in K \times Y:\left\langle G_{i} t_{i}, \eta_{i}\left(y_{i}, x_{i}\right)\right\rangle+S_{i}\left(x_{i}, y_{i}\right)\right. \\
& \left.\subseteq-\operatorname{int} C_{i}(x)\right\}
\end{aligned}
$$

is open and so $P_{i}$ has open lower sections.

For each $i \in I$, consider a set-valued mapping $Q_{i}: K \times Y \rightarrow 2^{K_{i}}$ defind by

$$
Q_{i}(x, t)=\operatorname{co}\left(P_{i}(x, t)\right) \cap D_{i}(x), \quad \forall(x, t) \in K \times Y .
$$

Since $D_{i}$ has open lower sections by hypothesis 1), we may apply Lemma 2.3 to assert that the set-valued mapping $Q_{i}$ has also open lower sections. Let

$$
W_{i}=\left\{(x, t) \in K \times Y: Q_{i}(x, t) \neq \varnothing\right\} \subset K \times Y .
$$

There are two cases to consider. In the case $W_{i}=\varnothing$, we have

$$
\operatorname{co}\left(P_{i}(x, t)\right) \cap D_{i}(x)=\varnothing, \forall(x, t) \in K \times Y .
$$

This implies that, $\forall(x, t) \in K \times Y$,

$$
P_{i}(x, t) \cap D_{i}(x)=\varnothing .
$$

On the other hand, by condition 1), and the fact $K_{i}$ is a compact convex subset of $E_{i}$, we can apply Lemma 2.4 to assert the existence of a fixed point $x_{i}^{*} \in D_{i}\left(x^{*}\right)$. Since $T_{i}\left(x^{*}\right) \neq \varnothing$, picking $t_{i}^{*} \in T_{i}\left(x^{*}\right)$, we have

$$
P_{i}\left(x^{*}, t^{*}\right) \cap D_{i}\left(x^{*}\right)=\varnothing \text {. }
$$

This implies $\forall y_{i} \in D_{i}\left(x^{*}\right), y_{i} \notin P_{i}\left(x^{*}, t^{*}\right)$. Hence, in this particular case, the assertion of the theorem holds.

We now consider the case $W_{i} \neq \varnothing$. Define a setvalued mapping $S_{i}: K \times Y \rightarrow 2^{K_{i}}$ by

$$
S_{i}(x, t)= \begin{cases}Q_{i}(x, t), & (x, t) \in W_{i} \\ D_{i}(x), & (x, t) \in K_{i} \times Y_{i} \backslash W_{i} .\end{cases}
$$

Then, $S_{i}(x, t)$ is a convex set-valued mapping and for each $u \in K, \quad S_{i}^{-1}(u)=Q_{i}^{-1}(u) \cup\left(D_{i}^{-1}(u) \times Y_{i}\right)$ is open. For each $i \in I$, consider the set-valued mapping $H: K \times Y \rightarrow 2^{K \times Y}$ where $H=\prod_{i \in I} H_{i}$ defined by

$$
H_{i}(x, t)=\left(S_{i}(x, t), T_{i}(x)\right) .
$$

By condition 1) and the properties of $S_{i}(x, t), H_{i}$ satisfies all the conditions of Lemma 2.4. Therefore, there exists $\left(x^{*}, t^{*}\right) \in K \times Y$ such that

$$
\begin{gathered}
\left(x_{i}^{*}, t_{i}^{*}\right) \in H_{i}\left(x^{*}, t^{*}\right) \text {. Suppose that }\left(x^{*}, t^{*}\right) \in W_{i} \text {, then } \\
x_{i}^{*} \in \operatorname{co}\left(P_{i}\left(x^{*}, t^{*}\right)\right) \cap D_{i}\left(x^{*}\right),
\end{gathered}
$$

so that $x_{i}^{*} \in \operatorname{co}\left(P_{i}\left(x^{*}, t^{*}\right)\right)$. This is a contradiction. Hence, $\left(x^{*}, t^{*}\right) \notin W_{i}$. Therefore,

$$
\left(x_{i}^{*}, t_{i}^{*}\right) \in\left(D_{i}\left(x^{*}\right), T_{i}\left(x^{*}\right)\right) \text {, and } Q_{i}\left(x^{*}, t^{*}\right)=\varnothing .
$$

Thus

$x_{i}^{*} \in D_{i}\left(x^{*}\right), t_{i}^{*} \in T_{i}\left(x^{*}\right), \quad \operatorname{co}\left(P_{i}\left(x^{*}, t^{*}\right)\right) \cap D_{i}\left(x^{*}\right)=\varnothing$.

This implies

$$
P_{i}\left(x^{*}, t^{*}\right) \cap D_{i}\left(x^{*}\right)=\varnothing .
$$

Consequently, the assertion of the theorem holds in this case.

Corollary 3.2. For each $i \in I$, let $Z_{i}$ be a 1.c.s., $K_{i}$ a nonempty compact convex subset of Hausdorff t.v.s. $E_{i}, Y_{i}$ a nonempty compact convex subset of $L\left(E_{i}, Z_{i}\right)$, which is equipped with a $\sigma$-topology. For each $i \in I$, assume that the following conditions are satisfied.

1) $D_{i}: K \rightarrow 2^{K_{i}}$ and $T_{i}: K \rightarrow 2^{Y_{i}}$ are two nonempty convex set-valued mappings and have open lower sections;

2) For all $y_{i} \in K_{i}$, the mapping

$\left\langle G_{i}, \eta_{i}\left(y_{i}, *\right)\right\rangle+S_{i}\left(*, y_{i}\right): K \times Y \rightarrow 2^{Z_{i}}$ is an u.s.c. setvalued mapping;

3) $C_{i}: K \rightarrow 2^{Z_{i}}$ is a convex set-valued mapping with int $C_{i}(x) \neq \varnothing$ for all $x \in K$;

4) $\eta_{i}: K_{i} \times K_{i} \rightarrow E_{i}$ is affine in the first argument and for all $x_{i} \in K_{i}, \eta_{i}\left(x_{i}, x_{i}\right)=0$;

5) $S_{i}: K \times K \rightarrow 2^{Z_{i}}$ is a generalized vector 0 -diagonally convex set-valued mapping;

6) For a given $x_{i} \in K_{i}$, and a neighborhood $U_{i}$ of $x$, for all $u \in U_{i}$, int $C_{i}(x)=\operatorname{int} C_{i}(u)$.

Then there exists $\bar{x}_{i} \in D_{i}(\bar{x})$ and $\bar{t}_{i} \in T_{i}(\bar{x})$ such that

$$
\left\langle G_{i} \bar{t}_{i}, \eta_{i}\left(y_{i}, \bar{x}_{i}\right)\right\rangle+S_{i}\left(\bar{x}_{i}, y_{i}\right) \not \subseteq-\operatorname{int} C_{i}(\bar{x}), \forall y_{i} \in D_{i}(\bar{x}) .
$$

Proof. Define a set-valued mapping $P_{i}: K \times Y \rightarrow 2^{K_{i}}$ by

$$
\begin{aligned}
& P_{i}(x, t)=\left\{y_{i} \in K_{i}:\left\langle G_{i} t_{i}, \eta_{i}\left(y_{i}, x_{i}\right)\right\rangle+S_{i}\left(x_{i}, y_{i}\right)\right. \\
&\left.\subseteq-\operatorname{int} C_{i}(x)\right\}, \\
& \forall(x, t) \in K \times Y .
\end{aligned}
$$

We first prove that $x_{i} \notin \operatorname{co}\left(P_{i}(x, t)\right)$ for all $(x, t) \in K \times Y$. By contradiction, for each $i \in I$, suppose there exists some point $(\bar{x}, \bar{t}) \in K \times Y$ such that $\bar{x}_{i} \in \operatorname{co}\left(P_{i}(\bar{x}, \bar{t})\right)$. Then, there exist finite points $y_{i_{1}}, y_{i_{2}}, \cdots, y_{i_{n}}$ in $K_{i}$, such that

$\left\langle G_{i} \bar{t}_{i}, \eta_{i}\left(y_{i}, \bar{x}_{i}\right)\right\rangle+S_{i}\left(\bar{x}_{i}, y_{i}\right) \subseteq-\operatorname{int} C_{i}(\bar{x}), i=1,2, \cdots, n$.

Since $\eta_{i}\left(., x_{i}\right)$ is affine and $\operatorname{int} C_{i}(\bar{x})$ is convex, for $\alpha_{j} \geq 0$ with $\sum_{j=1}^{n} \alpha_{j}=1$ such that $\bar{x}_{i}=\sum_{j=1}^{n} \alpha_{j} y_{i_{j}}$ and $y_{i_{j}} \in P_{i}(\bar{x}, \bar{t})$ for all $j=1, \cdots, n$ such that

$$
\begin{aligned}
& \left\langle G_{i} \bar{t}_{i}, \eta_{i}\left(\sum_{j=1}^{n} \alpha_{j} y_{i_{j}}, \bar{x}_{i}\right)\right\rangle+\sum_{j=1}^{n} \alpha_{j} S_{i}\left(\bar{x}_{i}, y_{i_{j}}\right) \subseteq-\operatorname{int} C_{i}(\bar{x}), \\
& j=1, \cdots, n .
\end{aligned}
$$


Since $\eta_{i}\left(x_{i}, x_{i}\right)=0$ for all $x_{i} \in K_{i}$

$$
\sum_{j=1}^{n} \alpha_{j} S_{i}\left(\bar{x}_{i}, y_{i}\right) \subseteq-\operatorname{int} C_{i}(\bar{x})
$$

which contradicts the hypothesis 5). Therefore $x_{i} \notin \operatorname{co}\left(P_{i}(x, t)\right)$.

We now prove that for each

$$
\begin{aligned}
& y_{i} \in K_{i}, P_{i}^{-1}\left(y_{i}\right) \\
= & \left\{(x, t) \in K \times Y:\left\langle G_{i} t_{i}, \eta_{i}\left(y_{i}, x_{i}\right)\right\rangle+S_{i}\left(x_{i}, y_{i}\right)\right. \\
& \left.\subseteq-\operatorname{int} C_{i}(x)\right\}
\end{aligned}
$$

is open. Indeed, let $(\bar{x}, \bar{t}) \in P_{i}^{-1}\left(y_{i}\right)$, that is

$\left\langle G_{i} \bar{t}_{i}, \eta_{i}\left(y_{i}, \bar{x}_{i}\right)\right\rangle+S_{i}\left(\bar{x}_{i}, y_{i}\right) \subseteq-\operatorname{int} C_{i}(\bar{x})$. Since

$\left\langle G_{i} t_{i}, \eta_{i}\left(y_{i},{ }^{*}\right)\right\rangle+S_{i}\left({ }^{*}, y_{i}\right): K \times Y \rightarrow 2^{Z_{i}}$ is an u.s.c. setvalued mapping, there exists a neighborhood $U_{i}$ of $(\bar{x}, \bar{t})$ such that

$\left\langle G_{i} t_{i}, \eta_{i}\left(y_{i}, x_{i}\right)\right\rangle+S_{i}\left(x_{i}, y_{i}\right) \subseteq-\operatorname{int} C_{i}(\bar{x}), \forall(x, t) \in U_{i}$.

By 6),

$\left\langle G_{i} t_{i}, \eta_{i}\left(y_{i}, x_{i}\right)\right\rangle+S_{i}\left(x_{i}, y_{i}\right) \subseteq-\operatorname{int} C_{i}(x), \forall(x, t) \in U_{i}$.

Hence, $U_{i} \subset P_{i}^{-1}\left(y_{i}\right)$. This implies, $P_{i}^{-1}\left(y_{i}\right)$ is open for each $y_{i} \in K_{i}$, and so $P_{i}$ have open lower sections. For the remainder of the proof, we can just follow that of Theorem 3.1. This completes the proof.

Corollary 3.3. For each $i \in I$, let $Z_{i}$ be a 1.c.s., $K_{i}$ a nonempty compact convex subset of Hausdorff t.v.s. $E_{i}, Y_{i}$ a nonempty compact convex subset of $L\left(E_{i}, Z_{i}\right)$, which is equipped with a $\sigma$-topology. For each $i \in I$, assume that the following conditions are satisfied.

1) $D_{i}: K \rightarrow 2^{K_{i}}$ and $T_{i}: K \rightarrow 2^{Y_{i}}$ are two nonempty convex set-valued mappings and have open lower sections;

2) For all $y_{i} \in K_{i}$, the mapping

$\left\langle G_{i}, \eta_{i}\left(y_{i},{ }^{*}\right)\right\rangle+S_{i}\left({ }^{*}, y_{i}\right): K \times Y \rightarrow 2^{Z_{i}}$ is an u.s.c. setvalued mapping;

3) $C_{i}: K \rightarrow 2^{Z_{i}}$ is a convex set-valued mapping such that for each $x \in K, C_{i}(x)=C_{i}$ is a convex cone with $\operatorname{int} C_{i}(x) \neq \varnothing$;

4) $\eta_{i}: K_{i} \times K_{i} \rightarrow E_{i}$ is affine in the first argument and for all $x_{i} \in K_{i}, \eta_{i}\left(x_{i}, x_{i}\right)=0$;

5) $S_{i}: K \times K \rightarrow 2^{Z_{i}}$ is a generalized vector 0 -diagonally convex set-valued mapping;

6) For a given $x_{i} \in K_{i}$, and a neighborhood $U_{i}$ of $x$, for all $u \in U_{i}, \quad \operatorname{int} C_{i}(x)=\operatorname{int} C_{i}(u)$.

Then there exist $\bar{x}_{i} \in D_{i}(\bar{x})$ and $\bar{t}_{i} \in T_{i}(\bar{x})$ such that

$$
\left\langle G_{i} \bar{t}_{i}, \eta_{i}\left(y_{i}, \bar{x}_{i}\right)\right\rangle+S_{i}\left(\bar{x}_{i}, y_{i}\right) \not \subseteq-\operatorname{int} C_{i}, \forall y_{i} \in D_{i}(\bar{x}) .
$$

Proof. By hypothesis 3), the condition 4) in Corollary 3.2 is satisfied. Hence, all the conditions are satisfied as in Corollary 3.2.

Corollary 3.4. For each $i \in I$, let $Z_{i}$ be a l.c.s., $K_{i}$ a nonempty compact convex subset of Hausdorff t.v.s. $E_{i}$, $Y_{i}$ a nonempty compact convex subset of $L\left(E_{i}, Z_{i}\right)$, which is equipped with a $\sigma$-topology. For each $i \in I$, assume that $S_{i}$ and $G_{i}$ are single valued mappings and the following conditions are satisfied.

1) $D_{i}: K \rightarrow 2^{K_{i}}$ and $T_{i}: K \rightarrow 2^{Y_{i}}$ are two nonempty convex set-valued mappings and have open lower sections;

2) For all $y_{i} \in K_{i}$, the mapping $\left\langle G_{i}, \eta_{i}\left(y_{i}, *\right)\right\rangle+S_{i}\left(*, y_{i}\right): K_{i} \times Y_{i} \rightarrow Z_{i}$ is continuous;

3) $C_{i}: K \rightarrow 2^{Z_{i}}$ is a convex set-valued mapping with int $C_{i}(x) \neq \varnothing$ for all $x \in K$;

4) $\eta_{i}: K_{i} \times K_{i} \rightarrow E_{i}$ is affine in the first argument and for all $x_{i} \in K_{i}, \eta_{i}\left(x_{i}, x_{i}\right)=0$;

5) $S_{i}: K \times K \rightarrow Z_{i}$ is a vector 0-diagonally convex mapping;

6) $Z_{i} \backslash\left\{-\operatorname{int} C_{i}(x)\right\}$ is an u.s.c. set-valued mapping.

Then there exist $\bar{x}_{i} \in D_{i}(\bar{x})$ and $\overline{t_{i}} \in T_{i}(\bar{x})$ such that

$$
\left\langle G_{i} \bar{t}_{i}, \eta_{i}\left(y_{i}, \bar{x}_{i}\right)\right\rangle+S_{i}\left(\bar{x}_{i}, y_{i}\right) \notin-\operatorname{int} C_{i}(\bar{x}), \forall y_{i} \in D_{i}(\bar{x}) .
$$

Proof. Define a set-valued mapping $P_{i}: K \times Y \rightarrow 2^{K_{i}}$ by

$$
\begin{aligned}
& P_{i}(x, t)=\left\{y_{i} \in K_{i}:\left\langle G_{i} t_{i}, \eta_{i}\left(y_{i}, x_{i}\right)\right\rangle+S_{i}\left(x_{i}, y_{i}\right)\right. \\
&\left.\in-\operatorname{int} C_{i}(x)\right\}, \\
& \forall(x, t) \in K \times Y .
\end{aligned}
$$

We now prove that for each

$$
\begin{aligned}
& y_{i} \in K_{i}, P_{i}^{-1}\left(y_{i}\right) \\
= & \left\{(x, t) \in K \times Y:\left\langle G_{i} t_{i}, \eta_{i}\left(y_{i}, x_{i}\right)\right\rangle+S_{i}\left(x_{i}, y_{i}\right)\right. \\
& \left.\in-\operatorname{int} C_{i}(x)\right\}
\end{aligned}
$$

is open, that is, the set

$$
\begin{aligned}
& \left\{(x, t) \in K \times Y:\left\langle G_{i} t_{i}, \eta_{i}\left(y_{i}, x_{i}\right)\right\rangle+S_{i}\left(x_{i}, y_{i}\right)\right. \\
& \left.\in Z_{i} \backslash\left\{-\operatorname{int} C_{i}(x)\right\}\right\}
\end{aligned}
$$

is closed. Indeed, let $\left\{\left(x^{o}, t^{o}\right)\right\}$ be a net in $K \times Y$ such that $\left(x^{o}, t^{o}\right) \rightarrow\left(x^{*}, t^{*}\right)$ and

$$
\left\langle G_{i} t_{i}^{o}, \eta_{i}\left(y_{i}, x_{i}^{o}\right)\right\rangle \cap S_{i}\left(x_{i}^{o}, y_{i}\right) \in Z_{i} \backslash\left\{-\operatorname{int} C_{i}\left(x^{o}\right)\right\} .
$$

Since $\left\langle G_{i}, \eta_{i}\left(y_{i},{ }^{*}\right)\right\rangle+S_{i}\left(*, y_{i}\right): K \times Y \rightarrow 2^{Z_{i}}$ is continuous, hence

$$
\begin{aligned}
& \left\langle G_{i} t_{i}^{o}, \eta_{i}\left(y_{i}, x_{i}^{o}\right)\right\rangle \cap S_{i}\left(x_{i}^{o}, y_{i}\right) \\
& \rightarrow\left\langle G_{i} t_{i}^{*}, \eta_{i}\left(y_{i}, x_{i}^{*}\right)\right\rangle+S_{i}\left(x_{i}^{*}, y_{i}\right) .
\end{aligned}
$$


Since $Z_{i} \backslash\left\{-\operatorname{int} C_{i}(x)\right\}$ is an u.s.c. set-valued mapping with closed values, by Lemma 2.1, we have

$$
\left\langle G_{i} t_{i}^{*}, \eta_{i}\left(y_{i}, x_{i}^{*}\right)\right\rangle+S_{i}\left(x_{i}^{*}, y_{i}\right) \in Z_{i} \backslash\left\{-\operatorname{int} C_{i}\left(x^{*}\right)\right\},
$$

and hence $\left(x^{*}, t^{*}\right)$ in the set

$$
\begin{aligned}
& \left\{(x, t) \in K \times Y:\left\langle G_{i} t_{i}, \eta_{i}\left(y_{i}, x_{i}\right)\right\rangle+S_{i}\left(x_{i}, y_{i}\right)\right. \\
& \left.\in-\operatorname{int} C_{i}(x)\right\} .
\end{aligned}
$$

This implies $P_{i}^{-1}\left(y_{i}\right)$ is open for each $y_{i} \in K_{i}$ and so $P_{i}$ has open lower sections. For the remainder of the proof, we can just follow that of Theorem 3.1 and Corollary 3.2. This completes the proof.

Theorem 3.5. For each $i \in I$, let $Z_{i}$ be a l.c.s., $K_{i}$ a nonempty compact convex subset of Hausdorff t.v.s. $E_{i}$, $Y_{i}$ a nonempty compact convex subset of $L\left(E_{i}, Z_{i}\right)$, which is equipped with a $\sigma$-topology. For each $i \in I$, assume that the following conditions are satisfied.

1) $D_{i}: K \rightarrow 2^{K_{i}}$ and $T_{i}: K \rightarrow 2^{Y_{i}}$ are two nonempty convex set-valued mappings and have open lower sections;

2) For each $t_{i} \in Y_{i}$ and $x_{i} \in \operatorname{co} \Lambda_{i}$, the mapping $\left\langle G_{i} t_{i}, \eta_{i}\left(., x_{i}\right)\right\rangle+S_{i}\left(x_{i},.\right): K \rightarrow 2^{Z_{i}}$ is WIC-DQC;

3) for each $y_{i} \in K_{i}$, the set

$$
\begin{aligned}
& \left\{(x, t) \in K \times Y:\left\{\left\langle G_{i} t_{i}, \eta_{i}\left(y_{i}, x_{i}\right)\right\rangle+S_{i}\left(x_{i}, y_{i}\right)\right\}\right. \\
& \left.\bigcap-\operatorname{int} C_{i}(x) \neq \varnothing\right\}
\end{aligned}
$$

is open.

Then there exist $\bar{x}_{i} \in D_{i}(\bar{x})$ and $\bar{t}_{i} \in T_{i}(\bar{x})$ such that

$$
\begin{aligned}
& \left\{\left\langle G_{i} \bar{t}_{i}, \eta_{i}\left(y_{i}, \bar{x}_{i}\right)\right\rangle+S_{i}\left(\bar{x}_{i}, y_{i}\right)\right\} \cap-\operatorname{int} C_{i}(\bar{x})=\varnothing, \\
& \forall y_{i} \in D_{i}(\bar{x}) .
\end{aligned}
$$

Proof. Define a set-valued mapping $P_{i}: K \times Y \rightarrow 2^{K_{i}}$ by

$$
\begin{aligned}
P_{i}(x, t)= & \left\{y_{i} \in K_{i}:\left\{\left\langle G_{i} t_{i}, \eta_{i}\left(y_{i}, x_{i}\right)\right\rangle+S_{i}\left(x_{i}, y_{i}\right)\right\}\right. \\
& \left.\bigcap-\operatorname{int} C_{i}(x) \neq \varnothing\right\}, \\
\forall(x, t) \in & K \times Y .
\end{aligned}
$$

For the remainder proof, we just follow that of Theorem 3.1 .

Corollary 3.6. For each $i \in I$, let $Z_{i}$ be a 1.c.s., $K_{i}$ a nonempty compact convex subset of Hausdorff t.v.s. $E_{i}$, $Y_{i}$ a nonempty compact convex subset of $L\left(E_{i}, Z_{i}\right)$, which is equipped with a $\sigma$-topology. For each $i \in I$, assume that the following conditions are satisfied.

1) $D_{i}: K \rightarrow 2^{K_{i}}$ and $T_{i}: K \rightarrow 2^{Y_{i}}$ are two nonempty convex set-valued mappings and have open lower sections;

2) For each $t_{i} \in Y_{i}$ and $x_{i} \in \operatorname{co} \Lambda_{i}$, the mapping $\left\langle G_{i} t_{i}, \eta_{i}\left(., x_{i}\right)\right\rangle+S_{i}\left(x_{i},.\right): K \rightarrow 2^{Z_{i}}$ is WIC-DQC;
3) $Z_{i} \backslash\left\{-\operatorname{int} C_{i}(x)\right\}$ is an u.s.c. set-valued mapping.

Then there exist $\bar{x}_{i} \in D_{i}(\bar{x})$ and $\overline{t_{i}} \in T_{i}(\bar{x})$ such that

$$
\begin{aligned}
& \left\{\left\langle G_{i} \bar{t}_{i}, \eta_{i}\left(y_{i}, \bar{x}_{i}\right)\right\rangle+S_{i}\left(\bar{x}_{i}, y_{i}\right)\right\} \cap-\operatorname{int} C_{i}(\bar{x})=\varnothing, \\
& \forall y_{i} \in D_{i}(\bar{x}) .
\end{aligned}
$$

Proof. Let $P_{i}: K \times Y \rightarrow 2^{K_{i}}$ be a set-valued mapping define in Theorem 3.5. We just prove that for each

$$
\begin{aligned}
& y_{i} \in K_{i}, P_{i}^{-1}\left(y_{i}\right) \\
= & \left\{(x, t) \in K \times Y:\left\{\left\langle G_{i} t_{i}, \eta_{i}\left(y_{i}, x_{i}\right)\right\rangle+S_{i}\left(x_{i}, y_{i}\right)\right\}\right. \\
& \left.\bigcap-\operatorname{int} C_{i}(x) \neq \varnothing\right\}
\end{aligned}
$$

is open, that is, the set

$$
\begin{aligned}
& \left\{(x, t) \in K \times Y:\left\{\left\langle G_{i} t_{i}, \eta_{i}\left(y_{i}, x_{i}\right)\right\rangle+S_{i}\left(x_{i}, y_{i}\right)\right\}\right. \\
& \left.\bigcap-\operatorname{int} C_{i}(x) \neq \varnothing\right\}
\end{aligned}
$$

is closed. Indeed, let $\left\{\left(x^{o}, t^{o}\right)\right\}$ be a net in $K \times Y$ such that $\left(x^{o}, t^{o}\right) \rightarrow\left(x^{*}, t^{*}\right)$ and

$$
\left\{\left\langle G_{i} t_{i}^{o}, \eta_{i}\left(y_{i}, x_{i}^{o}\right)\right\rangle+S_{i}\left(x_{i}^{o}, y_{i}\right)\right\} \cap-\operatorname{int} C_{i}\left(x^{o}\right)=\varnothing \text {. }
$$

This implies

$$
\left\{\left\langle G_{i} t_{i}^{o}, \eta_{i}\left(y_{i}, x_{i}^{o}\right)\right\rangle+S_{i}\left(x_{i}^{o}, y_{i}\right)\right\} \subseteq Z_{i} \backslash\left\{-\operatorname{int} C_{i}\left(x^{o}\right)\right\} .
$$

We now prove that

$$
\left\{\left\langle G_{i} t_{i}^{*}, \eta_{i}\left(y_{i}, x_{i}^{*}\right)\right\rangle+S_{i}\left(x_{i}^{*}, y_{i}\right)\right\} \subseteq Z_{i} \backslash\left\{-\operatorname{int} C_{i}\left(x^{*}\right)\right\} .
$$

If it is not true, then there exists a

$$
w^{*} \in\left\langle G_{i} t_{i}^{*}, \eta_{i}\left(y_{i}, x_{i}^{*}\right)\right\rangle+S_{i}\left(x_{i}^{*}, y_{i}\right) \text { such that }
$$

$w^{*} \notin Z_{i} \backslash\left\{-\operatorname{int} C_{i}\left(x^{*}\right)\right\}$. Since $Z_{i}$ is Hausdorff t.v.s.

(1.c.s. is Hausdorff space) and $Z_{i} \backslash\left\{-\operatorname{int} C_{i}\left(x^{*}\right)\right\}$ is closed, there exists two open sets $U_{i}, V_{i} \subset Z_{i}$ such that $w^{*} \in U_{i}, Z_{i} \backslash\left\{-\operatorname{int} C_{i}\left(x^{*}\right)\right\} \subset V_{i}$ and $U_{i} \cap V_{i}=\varnothing$.

Since $\left\langle G_{i}, \eta_{i}\left(y_{i},{ }^{*}\right)\right\rangle \cap S_{i}\left({ }^{*}, y_{i}\right): P \times Y \rightarrow 2^{Z_{i}}$ is an 1.s.c. set-valued mapping and $Z_{i} \backslash\left\{-\operatorname{int} C_{i}(x)\right\}$ is an u.s.c. set-valued mapping, there exists a neighborhood $U_{i}\left(x^{*}, y^{*}\right)$ of $\left(x^{*}, y^{*}\right)$ such that

$$
\begin{aligned}
& \left\{\left\langle G_{i}, \eta_{i}\left(y_{i}, x_{i}\right)\right\rangle+S_{i}\left(x_{i}, y_{i}\right)\right\} \cap U_{i} \neq \varnothing, \\
& \forall(x, t) \in U_{i}\left(x^{*}, y^{*}\right)
\end{aligned}
$$

and a neighborhood $U_{i}\left(x^{*}\right)$ of $x^{*}$ such that $Z_{i} \backslash\left\{-\operatorname{int} C_{i}(x)\right\} \subset V_{i}, \forall x \in U_{i}\left(x^{*}\right)$. 
Hence, for all $\left(x^{o}, t^{o}\right) \in U_{i}\left(x^{*}, y^{*}\right) \cap\left\{U_{i}\left(x^{*}\right) \times Y_{i}\right\}$, there exists $w^{o} \in\left\{\left\langle G_{i} t_{i}^{o}, \eta_{i}\left(y_{i}, x_{i}^{o}\right)\right\rangle+S_{i}\left(x_{i}^{o}, y_{i}\right)\right\}$ such that $w^{o} \notin Z_{i} \backslash\left\{-\operatorname{int} C_{i}\left(x^{o}\right)\right\}$, which is contradiction. Therefore, the set

$$
\begin{aligned}
& \left\{(x, t) \in K \times Y:\left\{\left\langle G_{i} t_{i}, \eta_{i}\left(y_{i}, x_{i}\right)\right\rangle+S_{i}\left(x_{i}, y_{i}\right)\right\}\right. \\
& \left.\bigcap-\operatorname{int} C_{i}(x)=\varnothing\right\}
\end{aligned}
$$

is closed. Hence, all the conditions of Theorem 3.5 satisfied. Consequently, the assertion of the theorem holds.

Theorem 3.7. For each $i \in I$, let $Z_{i}$ be a 1.c.s., $K_{i}$ a nonempty compact convex subset of Hausdorff t.v.s. $E_{i}$, $Y_{i}$ a nonempty compact convex subset of $L\left(E_{i}, Z_{i}\right)$, which is equipped with a $\sigma$-topology. For each $i \in I$, assume that the following conditions are satisfied.

1) $D_{i}: K \rightarrow 2^{K_{i}}$ and $T_{i}: K \rightarrow 2^{Y_{i}}$ are two nonempty convex set-valued mappings and have open lower sections;

2) For each $t_{i} \in Y_{i}$ and $x_{i} \in \operatorname{co} \Lambda_{i}$, the mapping $\left\langle G_{i} t_{i}, \eta_{i}\left(., x_{i}\right)\right\rangle+S_{i}\left(x_{i},.\right): K \rightarrow 2^{Z_{i}}$ is SIIC-DQC;

3) for each $y_{i} \in K_{i}$, the set

$$
\begin{aligned}
& \left\{(x, t) \in K \times Y:\left\{\left\langle G_{i} t_{i}, \eta_{i}\left(y_{i}, x_{i}\right)\right\rangle+S_{i}\left(x_{i}, y_{i}\right)\right\}\right. \\
& \left.\bigcap C_{i}(x)=\varnothing\right\}
\end{aligned}
$$

is open.

Then there exist $\bar{x}_{i} \in D_{i}(\bar{x})$ and $\overline{t_{i}} \in T_{i}(\bar{x})$ such that $\left\{\left\langle G_{i} \bar{t}_{i}, \eta_{i}\left(y_{i}, \bar{x}_{i}\right)\right\rangle+S_{i}\left(\bar{x}_{i}, y_{i}\right)\right\} \cap C_{i}(\bar{x}) \neq \varnothing, \forall y_{i} \in D_{i}(\bar{x})$.

Proof. Define a set-valued mapping $P_{i}: K \times Y \rightarrow 2^{K_{i}}$ by

$$
\begin{aligned}
& P_{i}(x, t)=\left\{y_{i} \in K_{i}:\left\{\left\langle G_{i} t_{i}, \eta_{i}\left(y_{i}, x_{i}\right)\right\rangle+S_{i}\left(x_{i}, y_{i}\right)\right\}\right. \\
&\left.\bigcap C_{i}(x)=\varnothing\right\}, \\
& \forall(x, t) \in K \times Y .
\end{aligned}
$$

For the remainder proof, we just follow that of Theorem 3.1 .

Corollary 3.8. For each $i \in I$, let $Z_{i}$ be a 1.c.s., $K_{i}$ a nonempty compact convex subset of Hausdorff t.v.s. $E_{i}$, $Y_{i}$ a nonempty compact convex subset of $L\left(E_{i}, Z_{i}\right)$, which is equipped with a $\sigma$-topology. For each $i \in I$, assume that the following conditions are satisfied.

1) $D_{i}: K \rightarrow 2^{K_{i}}$ and $T_{i}: K \rightarrow 2^{Y_{i}}$ are two nonempty convex set-valued mappings and have open lower sections; 2) For each $t_{i} \in Y_{i}$ and $x_{i} \in \operatorname{co} \Lambda_{i}$, the mapping $\left\langle G_{i} t_{i}, \eta_{i}\left(., x_{i}\right)\right\rangle+S_{i}\left(x_{i},.\right): K \rightarrow 2^{Z_{i}}$ is SIIC-DQC;

3) For all $x \in K, C_{i}(x)$ is closed convex cone $C_{i}$. Then there exist $\bar{x}_{i} \in D_{i}(\bar{x})$ and $\bar{t}_{i} \in T_{i}(\bar{x})$ such that

$$
\left\{\left\langle G_{i} \bar{t}_{i}, \eta_{i}\left(y_{i}, \bar{x}_{i}\right)\right\rangle+S_{i}\left(\bar{x}_{i}, y_{i}\right)\right\} \cap C_{i}=\varnothing, \forall y_{i} \in D_{i}(\bar{x}) .
$$

Proof. Let $P_{i}: K \times Y \rightarrow 2^{K_{i}}$ be a set-valued mapping defined in Theorem 3.7. We prove that for each

$$
\begin{aligned}
& y_{i} \in K_{i}, P_{i}^{-1}\left(y_{i}\right) \\
= & \left\{(x, t) \in K \times Y:\left\{\left\langle G_{i} t_{i}, \eta_{i}\left(y_{i}, x_{i}\right)\right\rangle+S_{i}\left(x_{i}, y_{i}\right)\right\}\right. \\
& \left.\bigcap C_{i}=\varnothing\right\}
\end{aligned}
$$

is open, that is, the set

$$
\left\{(x, t) \in K \times Y:\left\{\left\langle G_{i} t_{i}, \eta_{i}\left(y_{i}, x_{i}\right)\right\rangle+S_{i}\left(x_{i}, y_{i}\right)\right\} \subset Z_{i} \backslash C_{i}\right\}
$$

is open. If $(\bar{x}, \bar{t}) \in P_{i}^{-1}\left(y_{i}\right)$, since $Z_{i} \backslash C_{i}$ is open set and for all

$y_{i} \in K_{i},\left\langle G_{i}, \eta_{i}\left(y_{i}, *\right)\right\rangle+S_{i}\left(*, y_{i}\right): K \times Y \rightarrow 2^{Z_{i}}$, an u.s.c. set-valued mapping, there exists a neighborhood $U_{i}$ of $(\bar{x}, \bar{t})$, for all $(x, t) \in U_{i}$,

$$
\left\{\left\langle G_{i} t_{i}, \eta_{i}\left(y_{i}, x_{i}\right)\right\rangle+S_{i}\left(x_{i}, y_{i}\right)\right\} \subset Z_{i} \backslash C_{i} .
$$

This implies $P_{i}^{-1}\left(y_{i}\right)$ is open for each $y_{i} \in K_{i}$. Therefore, all the conditions of Theorem 3.7 are satisfied. Consequently the assertion of the theorem holds.

Theorem 3.9. For each $i \in I$, let $Z_{i}$ be a 1.c.s., $K_{i}$ a nonempty compact convex subset of Hausdorff t.v.s. $E_{i}$, $Y_{i}$ a nonempty compact convex subset of $L\left(E_{i}, Z_{i}\right)$, which is equipped with a $\sigma$-topology. For each $i \in I$, assume that the following conditions are satisfied.

1) $D_{i}: K \rightarrow 2^{K_{i}}$ and $T_{i}: K \rightarrow 2^{Y_{i}}$ are two nonempty convex set-valued mappings and have open lower sections;

2) For each $t_{i} \in Y_{i}$ and $x_{i} \in \operatorname{co} \Lambda_{i}$, the mapping $\left\langle G_{i} t_{i}, \eta_{i}\left(., x_{i}\right)\right\rangle+S_{i}\left(x_{i},.\right): K \rightarrow 2^{Z_{i}}$ is SIC-DQC;

3) for each $y_{j} \in K_{i}$, the set $\left\{(x, t) \in K \times Y:\left\langle G_{i} t_{i}, \eta_{i}\left(y_{i}, x_{i}\right)\right\rangle \cap S_{i}\left(x_{i}, y_{i}\right) \nsubseteq C_{i}(x)\right\} \quad$ is open.

Then there exist $\bar{x}_{i} \in D_{i}(\bar{x})$ and $\bar{t}_{i} \in T_{i}(\bar{x})$ such that

$$
\left\langle G_{i} \bar{t}_{i}, \eta_{i}\left(y_{i}, \bar{x}_{i}\right)\right\rangle+S_{i}\left(\bar{x}_{i}, y_{i}\right) \nsubseteq C_{i}(\bar{x}), \forall y_{i} \in D_{i}(\bar{x}) .
$$

Proof. Define a set-valued mapping $P_{i}: K \times Y \rightarrow 2^{K_{i}}$ by

$$
\begin{aligned}
& P_{i}(x, t)=\left\{y_{i} \in K_{i}:\left\langle G_{i} t_{i}, \eta_{i}\left(y_{i}, x_{i}\right)\right\rangle+S_{i}\left(x_{i}, y_{i}\right) \nsubseteq C_{i}(x)\right\}, \\
& \forall(x, t) \in K \times Y .
\end{aligned}
$$

The rest of the proof is similar to that of Theorem 3.1.

Corollary 3.10. For each $i \in I$, let $Z_{i}$ be a l.c.s., $K_{i}$ a nonempty compact convex subset of Hausdorff t.v.s. $E_{i}, Y_{i}$ a nonempty compact convex subset of $L\left(E_{i}, Z_{i}\right)$, which is equipped with a $\sigma$-topology. For each $i \in I$, assume that the following conditions are satisfied.

1) $D_{i}: K \rightarrow 2^{K_{i}}$ and $T_{i}: K \rightarrow 2^{Y_{i}}$ are two nonempty convex set-valued mappings and have open lower sections; 2) For each $t_{i} \in Y_{i}$ and $x_{i} \in \operatorname{co} \Lambda_{i}$, the mapping 
$\left\langle G_{i} t_{i}, \eta_{i}\left(., x_{i}\right)\right\rangle+S_{i}\left(x_{i},.\right): K \rightarrow 2^{Z_{i}}$ is SIC-DQC;

3) $C_{i}(x)$ is an u.s.c. mapping with closed values.

Then there exist $\bar{x}_{i} \in D_{i}(\bar{x})$ and $\bar{t}_{i} \in T_{i}(\bar{x})$ such that

$$
\left\langle G_{i} \bar{t}_{i}, \eta_{i}\left(y_{i}, \bar{x}_{i}\right)\right\rangle+S_{i}\left(\bar{x}_{i}, y_{i}\right) \subseteq C_{i}(\bar{x}), \forall y_{i} \in D_{i}(\bar{x}) .
$$

Proof. Let $P_{i}: K \times Y \rightarrow 2^{K_{i}}$ a set-valued mapping defined in Theorem 3.9. We prove that for each $y_{i} \in K_{i}$, the set

$\left\{(x, t) \in K \times Y:\left\langle G_{i} t_{i}, \eta_{i}\left(y_{i}, x_{i}\right)\right\rangle \cap S_{i}\left(x_{i}, y_{i}\right) \not C_{i}(x)\right\} \quad$ is open, that is, the set

$\left\{(x, t) \in K \times Y:\left\langle G_{i} t_{i}, \eta_{i}\left(y_{i}, x_{i}\right)\right\rangle \cap S_{i}\left(x_{i}, y_{i}\right) \subseteq C_{i}(x)\right\} \quad$ is closed. Indeed, let $\left\{\left(x^{o}, t^{o}\right)\right\}$ be a net in $K \times Y$ such that $\left(x^{o}, t^{o}\right) \rightarrow\left(x^{*}, t^{*}\right)$ and

$$
\left\langle G_{i} t_{i}^{o}, \eta_{i}\left(y_{i}, x_{i}^{o}\right)\right\rangle \cap S_{i}\left(x_{i}^{o}, y_{i}\right) \subseteq C_{i}\left(x^{o}\right) .
$$

We claim that

$$
\left\langle G_{i} t_{i}^{*}, \eta_{i}\left(y_{i}, x_{i}^{*}\right)\right\rangle \cap S_{i}\left(x_{i}^{*}, y_{i}\right) \subseteq C_{i}\left(x^{*}\right) .
$$

To prove this assertion, we can just follow that of Corollary 3.6. Hence, the set

$\left\{(x, t) \in K \times Y:\left\langle G_{i} t_{i}, \eta_{i}\left(y_{i}, x_{i}\right)\right\rangle \cap S_{i}\left(x_{i}, y_{i}\right) \not C_{i}(x)\right\} \quad$ is open. Therefore, all the conditions of Theorem 3.9 are satisfied. Consequently, the assertion of the corollary hold.

\section{REFERENCES}

[1] J.-W. Peng, "System of Generalized Set-Valued QuasiVariational-Like Inequalities," Bulletin of the Australian Mathematical Society, Vol. 68, No. 3, 2003, pp. 501-515. doi:10.1017/S0004972700037904

[2] Q. H. Ansari, S. Schaible and J. C. Yao, "The System of Generalized Vector Equilibrium Problems with Applications," Journal of Global Optimization, Vol. 22, No. 1, 2002, pp. 3-16. doi:10.1023/A:1013857924393

[3] H. H. Schaefer, "Topological Vector Space," 2nd Edition, Graduate Texts in Mathematics, Springer, Berlin, 1999.

[4] X. P. Ding and E. Tarafdar, "Generalized VariationalLike Inequalities with Pseudomonotone Set-Valued Mappings," Archive der Mathematik, Vol. 74, No. 4, 2000, pp. 302-313. doi:10.1007/s000130050447

[5] Q. H. Ansari and J. C. Yao, "System of Generalized Varitional Ineqalities and Their Applications," Applicable Analysis, Vol. 76, No. 3-4, 2000, pp. 203-217. doi:10.1080/00036810008840877

[6] G. Xiao and S. Liu, "Existence of Solutions for General- ized Vector Quasi-Variational-Like Inequalities without Monotonicity," Computers and Mathematics with Applications, Vol. 58, No. 8, 2009, pp. 1550-1557. doi:10.1016/j.camwa.2009.05.021

[7] G. Xiao, Z. Fan and X. Qi, "Existence Results for Generalized Nonlinear Vector Variational-Like Inequalities with Set-Valued Mapping," Applied Mathematics Letter, Vol. 23, No. 1, 2010, pp. 44-47. doi:10.1016/j.aml.2009.07.023

[8] J.-W. Peng and X.-M. Yang, "Generalized Vector QuasiVariational-Like Inequalities," Journal of Inequalities and Applications, Vol. 2006, No. 1, 2006, pp. 1-11.

[9] S. Husain and S. Gupta, "Existence of Solutions for Generalized Nonlinear Vector Quasi-Variational-Like Inequalities with Set-Valued Mappings," Filomat, Vol. 26, No. 5, 2012, pp. 909-916. doi:10.2298/FIL1205909H

[10] S. Husain and S. Gupta, "Generalized Nonlinear Vector Quasi-Variational-Like Inequality," International Journal of Pure and Applied Mathematics, Vol. 66, No. 2, 2011, pp. 157-169.

[11] P. Hartman and G. Stampacchia, "On Some Nonlinear Elliptic Differential Function Equations," Acta Mathematica, Vol. 115, No. 1, 1966, pp. 271-310. doi:10.1007/BF02392210

[12] S. H. Hou, H. Yu and G. Y. Chen, "On Vector QuasiEquilibrium Problems with Set-Valued Maps," Journal of Optimization Theory and Applications, Vol. 119, No. 3, 2003, pp. 485-498. doi:10.1023/B:JOTA.0000006686.19635.ad

[13] Q. M. Liu, L. Y. Fan and G. H. Wang, "Generalized Vector Quasi-Equilibrium Problems with Set-Valued Mappings," Applied Mathematics Letters, Vol. 21, No. 9, 2008, pp. 946-950. doi:10.1016/j.aml.2007.10.007

[14] G. Q. Tian and J. Zhou, "Quasi-Variational Inequalities without the Concavity Assumption," Journal of Mathematical Analysis and Applications, Vol. 172, No. 1, 1993, pp. 289-299. doi:10.1006/jmaa.1993.1025

[15] J. P. Aubin and I. Ekeland, "Applied Nonlinear Analysis," Wiley-Interscience, New York, 1984.

[16] C. H. Su and V. M. Sehgal, "Some Fixed Point Theorems for Condensing Multifunctions in Locally Convex Spaces," Proceedings of the American Mathematical Society, Vol. 50, No. 1, 1975, pp. 150-154. doi:10.1090/S0002-9939-1975-0380530-7

[17] X. Q. Yang, "Generalized Convex Functions and Vector Variational Inequalities," Journal of Optimization Theory and Applications, Vol. 79, No. 3, 1993, pp. 563-580. doi:10.1007/BF00940559

[18] Q. H. Ansari and J. C. Yao, "A Fixed Point Theorem and Its Applications to the System of varItional Ineqalities," Bulletin of the Australian Mathematical Society, Vol. 59, No. 3, 1999, pp. 433-442. doi:10.1017/S0004972700033116 